\title{
EFEITO IN VITRO DO BARBATIMÃO EM MARCADORES DE ESTRESSE OXIDATIVO DE FIBROBLASTOS HUMANOS SENSCENTES
}

Nathália Cardoso de Afonso Bonotto; Laboratório de Biogenômica, Departamento de Morfologia, Universidade Federal de Santa Maria; nathaaliab23@gmail.com

Moisés Henrique Mastella; Programa de Pós-Graduação em Gerontologia, Universidade Federal de Santa Maria; mhmastella@gmail.com Ivana Beatrice Mânica da Cruz; Programa de Pós-Graduação em Gerontologia, Universidade Federal de Santa Maria; ibmcruz@hotmail.com

Neida Luiza Caspary Pellenz; Programa de Pós-Graduação em Farmacologia, Universidade Federal de Santa Maria; neidaluiza@gmail.com

Euler Esteves Ribeiro; Fundação Universidade Aberta da Terceira Idade; unatieuler@gmail.com Verônica Farina Azzolin; Fundação Universidade Aberta da Terceira Idade - azzolinveronica@gmail.com Fernanda Barbisan; Programa de Pós-Graduação em Gerontologia, Universidade Federal de Santa Maria; fernandabarbisan@gmail.com

\section{RESUMO}

Introdução: Os fibroblastos compõem as principais células da pele, sendo responsáveis pelo processo de cicatrização de lesões. O estresse oxidativo, causado pela produção excessiva de Espécies Reativas de Oxigênio (EROs), é responsável por agravar o processo de envelhecimento celular, prejudicando as funções de proliferação e cicatrização dos fibroblastos, contribuindo para o aparecimento de lesões teciduais. Desta forma, torna-se relevante a busca por compostos naturais que possam auxiliar no reparo destas lesões, como é o caso do barbatimão, planta do Cerrado, utilizada pela medicina tradicional como cicatrizante devido à sua potencial atividade antioxidante. Objetivo: Avaliar in vitro o efeito do barbatimão em marcadores de estresse oxidativo de fibroblastos humanos senescentes. Métodos: Células da linhagem de fibroblastos humanos (HFF-1) foram cultivadas em condições controladas até atingirem morfologia senescente via observação por microscopia confocal. As células senescentes foram expostas ao extrato de barbatimão nas concentrações de $0,49 \mathrm{mg} / \mathrm{mL}$ e $0,99 \mathrm{mg} / \mathrm{mL}$. Após 72 horas de exposição, foram analisados espectrofotometricamente os níveis de carbonilação de proteínas e lipoperoxidação. Resultados: O barbatimão mostrou-se eficiente em reduzir os níveis dos marcadores oxidativos em ambas as concentrações utilizadas, diminuindo tanto o dano às proteínas como aos lipídeos. Conclusões: Apesar das limitações metodológicas inerentes aos estudos in vitro, nossos resultados indicam que o barbatimão desempenha atividade positiva no combate ao estresse oxidativo, podendo ser um poderoso aliado na prevenção do aparecimento de lesões por pressão, um grande problema de saúde pública no Brasil, principalmente entre idosos acamados. Entretanto, mais estudos precisam ser realizados para confirmação desta hipótese.

Palavras-chave: Envelhecimento; Senescência celular; Espécies reativas de oxigênio. 